\title{
PLANTAS MEDICINALES HIPOTENSORAS Y SUS PRODUCTOS COMERCIALIZADOS EN EL ÁREA METROPOLITANA DE BUENOS AIRES, ARGENTINA
}

\author{
Hypotensives medicinal plants and their products commercialized in the Metropolitan Area of \\ Buenos Aires, Argentina
}

JEREMIAS P. PUENTES ${ }^{1}$ iD

\begin{abstract}
Resumen: Esta contribución se enmarca dentro de una línea de investigación en Etnobotánica urbana, la cual estudia el conocimiento botánico en áreas urbanas. Se incluye el registro de 83 plantas medicinales que se comercializan como hipotensoras en el Área Metropolitana de Buenos Aires. Los datos sobre los usos locales asignados de las plantas se obtuvieron del trabajo de campo a través de metodologías etnobotánicas cualitativas. Se relevaron 180 sitios de expendio: 145 locales del circuito comercial general (farmacias, herboristerías, dietéticas), y 35 de los circuitos comerciales restringidos de dos segmentos de inmigrantes, chinos y bolivianos: el "Mercado Boliviano" del barrio de Liniers, con 30 sitios de expendio: locales y puestos callejeros, y el denominado "Barrio Chino", un sector del barrio de Belgrano con cinco grandes supermercados. Se realizó una búsqueda bibliográfica sobre los estudios académicos vinculados a la hipertensión con el objetivo de correlacionar el uso asignado. De las 83 especies relevadas, $10(12 \%)$ son invisibles para la mayoría de la población urbana local, mientras que las $73(88 \%)$ restantes son visibles. Se aportan datos etnobotánicos sobre plantas vinculadas al tratamiento de la hipertensión y la validez científica de su uso, información adecuada para los consumidores que deciden emplear esas plantas.
\end{abstract}

Palabras clave: Conocimiento botánico, etnobotánica urbana, hipotensores.

Summary: This study is part of a research project in Urban Ethnobotany, which studies the botanical knowledge in urban areas. It includes 83 medicinal plants marketed as hypotensive in the Metropolitan Area of Buenos Aires. Data on the local use of these plants were gathered via field work and qualitative ethnobotanical methodologies. The 180 outlets surveyed consisted of: 145 stores of the general commercial circuit (pharmacies, herbalists, dietetics) and 35 outlets of the restricted commercial circuits of two immigrants segments, Chinese and Bolivians. The "Bolivian Market" of the Liniers district included 30 outlets (local and street stalls). The so-called "Chinatown", a sector of the Belgrano neighborhood, included five large supermarkets. A search of academic studies related to hypertension was carried out to correlate the assigned plant use. Of the 83 species surveyed, $10(12 \%)$ are invisible to most of the local urban population, while the remaining $73(88 \%)$ are visible. Ethnobotanical information is provided on plants linked to hypertension treatment and on its scientific validity, adapting it to consumers who decide to use these plants.

Key words: Botanical knowledge, hypotensive, urban ethnobotany.

\footnotetext{
${ }^{1}$ Laboratorio de Etnobotánica y Botánica Aplicada (LEBA), Facultad de Ciencias Naturales y Museo, Universidad Nacional de La Plata. Calle 64 nro. 3, 1900-La Plata, Buenos Aires. E-mail: jeremiasppuentes@gmail.com
} 


\section{Introducción}

El presente trabajo forma parte de una línea de investigación en Etnobotánica urbana desarrollada por el Laboratorio de Etnobotánica y Botánica Aplicada (LEBA) en el Área Metropolitana de Buenos Aires (AMBA), que estudia las complejas relaciones entre las personas y su entorno vegetal en contextos pluriculturales urbanos, en especial la composición y dinámica del conocimiento botánico local (Hurrell, 2014; Hurrell \& Pochettino, 2014; Puentes, 2017).

El marco teórico-metodológico asume que este conocimiento no es accesible de manera directa, pero puede extrapolarse de las "acciones" que este conocimiento orienta: discursos, estrategias de selección, uso y consumo de plantas y sus productos. El conocimiento botánico (CB) constituye un conjunto complejo de conocimientos y creencias sobre las plantas, sus partes y productos derivados, y tiene dos componentes: 1) componentes no tradicionales: el conocimiento enseñado en la educación formal y el difundido a través de los medios, incluido el conocimiento científico; 2) componentes ligados a tradiciones: las tradiciones familiares locales de larga data y las de distintos grupos de inmigrantes, de diverso origen y tiempo de permanencia en el área de estudio. La dinámica del CB local se evidencia a través de la circulación de los productos en los circuitos comerciales restringidos de los grupos de inmigrantes y en el circuito comercial general.

Los productos vegetales comercializados en dichos circuitos propios de los inmigrantes pueden permanecer "invisibles" para la mayor parte de la población local, o bien ingresar al circuito comercial general, donde se tornan "visibles". El pasaje de plantas y productos desde el circuito restringido de los inmigrantes hacia el circuito comercial general se ha denominado "proceso de visualización" (Hurrell, 2014). Este proceso involucra diferentes agentes de visualización, en especial, los sitios de expendio llamados "dietéticas", que expenden alimentos saludables, nutracéuticos, fitoterápicos, suplementos dietéticos, y los medios masivos de comunicación (principalmente Internet), que potencian la transmisión del $\mathrm{CB}$ de forma rápida y en múltiples direcciones a la vez (Arenas et al., 2011, 2015; Pochettino \& Hurrell, 2013; Hurrell et al., 2013, 2015 a, b; Hurrell \& Pochettino, 2014; Puentes \& Hurrell, 2015; Cristina, 2016; Puentes, 2016, 2017). Este trabajo se focaliza en las plantas medicinales localmente identificadas y empleadas como hipotensoras, sus saberes asociados, y su visibilidad a partir de su circulación en los circuitos comerciales del AMBA.

La "hipertensión", "tensión arterial alta" o "elevada", es un trastorno en el que los vasos sanguíneos tienen una tensión alta persistente que puede dañarlos (Pérez \& Unanua, 2002). La hipertensión es considerada una de las principales causantes de las afecciones cardiovasculares (OMS, 2019). En el mundo, las enfermedades cardiovasculares son responsables de aproximadamente 17 millones de muertes por año, casi un tercio del total. Las complicaciones de la hipertensión causan anualmente 9,4 millones de muertes. La hipertensión es la causa de por lo menos el $45 \%$ de las muertes por cardiopatías y el $51 \%$ de las muertes por accidente cerebrovascular (OMS, 2013). Las consecuencias adversas de la hipertensión son complejas porque muchos afectados tienen además otros factores de riesgo que aumentan la probabilidad de infarto de miocardio, accidente cerebrovascular e insuficiencia renal. Entre esos factores de riesgo se encuentran el consumo de tabaco, la obesidad, el hipercolesterolemia y la diabetes mellitus (Lara et al., 2004).

Estas enfermedades son producto de la alimentación poco saludable y el ritmo acelerado característicos del estilo de vida urbano (Arenas et al., 2011; Hurrell et al., 2015b, Puentes, 2016), además de tener un fuerte componente genético (Miranda et al., 2012). En este contexto, muchos habitantes locales recurren al empleo de medicinas alternativas. Esta problemática ha impulsado a realizar trabajos etnobotánicos en el AMBA, sobre la calidad de los productos vegetales hipotensores (Cortella \& Pochettino, 1999), y sobre el CB local acerca de las plantas medicinales utilizadas como adelgazantes, potenciadores cognitivos, hipocolesterolémicos y antidiabéticos (Arenas et al., 2013, 2015; Hurrell et al., 2015a, b; Molares et al., 2012, Puentes, 2016). El objetivo principal de este trabajo es aportar a 
la caracterización y estudio del conocimiento botánico urbano, a través del análisis de los productos de plantas medicinales hipotensoras que se comercializan en el AMBA, junto a sus saberes asociados. Asimismo, se aportan datos sobre los efectos estudiados y la actividad biológica, consultados en la bibliografía disponible, a fin de compararlos con el uso hipotensor localmente asignado.

\section{Materiales y Métodos}

El área de estudio corresponde al Área Metropolitana Buenos Aires (AMBA), que comprende dos aglomerados urbanos contiguos: el Gran Buenos Aires y el Gran La Plata. El primero incluye la Ciudad Autónoma de Buenos Aires, la capital de la Argentina, $\mathrm{y}$ distritos vecinos de la provincia de Buenos Aires, en total, 3850 kilómetros cuadrados y unos 13 millones de habitantes en 2010 (INDEC 2010). En la ciudad de Buenos Aires viven unos 3 millones de personas en solo 203 kilómetros cuadrados. Este aglomerado urbano es el más grande en extensión y población de la Argentina, el segundo de Sudamérica, el tercero de América Latina, el quinto de América y el decimoséptimo del mundo (Forstall et al., 2004). El Gran La Plata incluye la ciudad de La Plata, capital de la provincia de Buenos Aires, y los distritos vecinos de Ensenada y Berisso, tiene 1150 kilómetros cuadrados y su población es de unos 800.000 habitantes (INDEC 2010).

Los datos sobre los usos localmente asignados de las plantas y productos que circulan en el ámbito urbano se obtuvieron del trabajo de campo etnobotánico, realizado sistemáticamente en el marco de la línea de investigación del LEBA desde 2005. El uso hipotensor presenta un amplio consenso entre los informantes y se corresponde asimismo con la información proveniente de otras fuentes, como la de etiquetas, prospectos y catálogos, impresos y electrónicos. La información también se complementó con datos difundidos en Internet, que orienta la selección de productos vegetales por parte de numerosos consumidores urbanos que confían en esa fuente de información. La búsqueda se orientó hacia las plantas medicinales hipotensoras, por uso y nombres científicos y vernáculos, hasta alcanzar la saturación de la información requerida. De este modo, el uso localmente asignado (hipotensor) es una categoría compleja que se construye a partir de la información de las distintas fuentes antes mencionadas, al igual que en el caso de las plantas hipocolesterolémicas y antidiabéticas (Hurrell et al., 2015b; Puentes, 2016). Al trabajo de campo se sumó una revisión de la bibliografía académica disponible para cada especie, de modo de obtener datos clínicos y de laboratorio acerca de los efectos y actividad biológica vinculados a la disminución de la presión arterial, para evaluar su correspondencia con los usos localmente asignados. Entre los agentes que disminuyen la presión arterial, llamados hipotensores o antihipertensores, también se encuentran los diuréticos y los vasodilatadores/vasorelajadores, entre otros (Hoffman, 2007).

En los relevamientos se emplearon metodologías etnobotánicas cualitativas habituales: observación participante, listados libres, entrevistas abiertas y semiestructuradas (Quinlan, 2005; Etkin \& Ticktin, 2010; Albuquerque et al., 2014). Se relevaron 180 sitios de expendio: 145 locales del circuito comercial general (farmacias, herboristerías, dietéticas), y 35 sitios de expendio de los circuitos comerciales restringidos de dos segmentos de inmigrantes, chinos y bolivianos, localizados en la Ciudad Autónoma de Buenos Aires. Estos segmentos de inmigrantes introducen y comercializan plantas y productos de derivados relacionados con sus tradiciones de origen, en dos lugares específicos: el "Mercado Boliviano" del barrio de Liniers, con 30 sitios de expendio: locales y puestos callejeros, y el denominado "Barrio Chino", un sector del barrio de Belgrano con cinco grandes supermercados (Arenas et al., 2011; Pochettino et al., 2012; Hurrell et al., 2013; Hurrell \& Puentes, 2013, 2017; Hurrell, 2014; Hurrell \& Pochettino, 2014; Puentes, 2016). La selección de los sitios de expendio del circuito comercial general comenzó al azar y finalizó con la saturación de la información sobre las especies y productos. Se entrevistaron dos informantes por cada sitio de expendio, 360 personas en total, previo consentimiento informado. Cerca del $80 \%$ de los entrevistados corresponde a vendedores de ambos sexos y de entre 18 y 65 años, quienes conocen las propiedades y beneficios de los productos que 
venden y guían a los consumidores sobre sus formas de empleo y administración; el resto de las personas entrevistadas corresponde a consumidores locales.

En los relevamientos realizados se obtuvieron muestras de referencia que fueron depositadas en las colecciones etnobotánicas del LEBA, cada una identificadas con un código alfanumérico (marcado entre corchetes). La identificación de las muestras se realizó mediante la evaluación de caracteres morfológicos macroscópicos y, cuando fue necesario, se realizó un análisis micrográfico de materiales fragmentados y pulverizados (Cuassolo et al., 2010; Gurni, 2014). El tratamiento taxonómico de las especies se ha ajustado según las bases de datos: The Plant List (2013), IBODA (2019) y Tropicos.org (2019).

\section{Resultados}

Esta contribución presenta 83 especies medicinales y sus productos derivados, difundidos, identificados y consumidos como hipotensores en el área de estudio. En la Tabla 1 se indican nombres científicos, familias botánicas, nombres vernáculos, tipos de productos comercializados y muestras de referencia (entre corchetes), y la actividad biológica y efectos estudiados. Las muestras con un asterisco (*) pertenecen al "Mercado Boliviano" del barrio porteño de Liniers; las que se indican con dos asteriscos (**) al "Barrio Chino" de Belgrano, Capital Federal; y las que no presentan asterisco corresponden al circuito comercial general.

Tabla 1. Plantas medicinales hipotensoras y productos comercializados en la Área Metropolitana de Buenos Aires, Argentina.

Table 1. Hypotensive medicinal plants and products marketed in the Metropolitan Area of Buenos Aires, Argentina.

\begin{tabular}{|c|c|c|}
\hline $\begin{array}{l}\text { Especies, familias, nombres } \\
\text { vulgares }\end{array}$ & Productos [Muestras] & $\begin{array}{c}\text { Actividad biológica y efectos } \\
\text { estudiados }\end{array}$ \\
\hline $\begin{array}{l}\text { Acacia senegal (L.) Willd. } \\
\text { FABACEAE } \\
\text { Goma arábiga }\end{array}$ & $\begin{array}{l}\text { Material pulverizado a granel } \\
\text { [AC1102] }\end{array}$ & Sin datos. \\
\hline $\begin{array}{l}\text { Achillea millefolium } \mathrm{L} \text {. } \\
\text { ASTERACEAE } \\
\text { Milenrama }\end{array}$ & $\begin{array}{l}\text { Partes aéreas secas } \\
\text { envasadas [C001] } \\
\text { Tintura madre [H348] }\end{array}$ & $\begin{array}{l}\text { Hipotensor, diurético (Alonso \& } \\
\text { Desmarchelier, 2005; de Souza et } \\
\text { al., 2011, 2013; Ali et al., 2017). }\end{array}$ \\
\hline $\begin{array}{l}\text { Adonis vernalis } \mathrm{L} \text {. } \\
\text { RANUNCULACEAE } \\
\text { Adonis }\end{array}$ & $\begin{array}{l}\text { Partes aéreas secas } \\
\text { fragmentadas envasadas } \\
\text { [D145] }\end{array}$ & Sin datos. \\
\hline $\begin{array}{l}\text { Allium sativum L. } \\
\text { AMARYLLIDACEAE } \\
\text { Ajo }\end{array}$ & Comprimidos [H329] & $\begin{array}{l}\text { Hipotensor, diurético (Brändle et } \\
\text { al., 1997; Alonso \& Desmarchelier, } \\
\text { 2005; Singh \& Singh, 2008; } \\
\text { Brankovic et al., 2011; Nwokocha et } \\
\text { al., 2011; Bhandari, 2012; Chaupis- } \\
\text { Meza et al., 2014). }\end{array}$ \\
\hline $\begin{array}{l}\text { Allophylus edulis (A. St.-Hil., } \\
\text { A. Juss. \& Cambess.) Hieron. ex } \\
\text { Niederl. } \\
\text { SAPINDACEAE } \\
\text { Cocú }\end{array}$ & $\begin{array}{l}\text { Partes aéreas secas } \\
\text { fragmentadas envasadas } \\
{[\mathrm{H} 145]}\end{array}$ & Sin datos. \\
\hline $\begin{array}{l}\text { Aloysia citrodora Palau } \\
\text { VERBENACEAE } \\
\text { Cedrón, yerba luisa }\end{array}$ & $\begin{array}{l}\text { Hojas secas a granel [C009] } \\
\text { Té en saquitos [H044] } \\
\text { Té en saquitos (mezcla) } \\
\text { [H047] } \\
\text { Tintura madre [R102] }\end{array}$ & $\begin{array}{l}\text { Hipotensor (Ragone et al., 2010; } \\
\text { Hurrell, 2018). }\end{array}$ \\
\hline $\begin{array}{l}\text { Angelica sinensis (Oliv.) Diels } \\
\text { APIACEAE } \\
\text { Dang gui, angélica china }\end{array}$ & $\begin{array}{l}\text { Raíces secas fragmentadas } \\
\text { envasadas }[\mathrm{H} 399]^{* *}\end{array}$ & $\begin{array}{l}\text { Hipotensor, vasodilatador (Puentes, } \\
\text { 2017). }\end{array}$ \\
\hline
\end{tabular}




\begin{tabular}{|c|c|c|}
\hline $\begin{array}{l}\text { Especies, familias, nombres } \\
\text { vulgares }\end{array}$ & Productos [Muestras] & $\begin{array}{l}\text { Actividad biológica y efectos } \\
\text { estudiados }\end{array}$ \\
\hline $\begin{array}{l}\text { Annona muricata L. } \\
\text { ANNONACEAE } \\
\text { Graviola }\end{array}$ & $\begin{array}{l}\text { Hojas secas a granel }[\mathrm{H} 304] \\
\text { Tintura madre }[\mathrm{H} 324] \\
\text { Cápsulas }[\mathrm{H} 288]^{* *}[\mathrm{H} 292]\end{array}$ & $\begin{array}{l}\text { Hipotensor (Nwokocha et al., } \\
\text { 2012; Adefegha et al., 2015; } \\
\text { Moghadamtousi et al., 2015; } \\
\text { Puentes, 2017; Coria Tellez et al., } \\
\text { 2018). }\end{array}$ \\
\hline $\begin{array}{l}\text { Artemisia absinthium L. } \\
\text { ASTERACEAE } \\
\text { Ajenjo }\end{array}$ & $\begin{array}{l}\text { Plantas frescas a granel } \mathrm{H} 165]^{*} \\
\text { Partes aéreas secas } \\
\text { fragmentadas envasadas } \\
\text { [P144] } \\
\text { Tintura madre [H356] }\end{array}$ & $\begin{array}{l}\text { Vasodilatador, diurético (Alonso \& } \\
\text { Desmarchelier, 2005). }\end{array}$ \\
\hline $\begin{array}{l}\text { Astragalus mongholicus Bunge } \\
\text { [= A. membranaceus Bunge] } \\
\text { FABACEAE } \\
\text { Astrágalo }\end{array}$ & $\begin{array}{l}\text { Raíces secas fragmentadas } \\
\text { envasadas [P183] [H400] }{ }^{* *} \\
\text { Cápsulas (mezcla) }[\mathrm{H} 323]\end{array}$ & $\begin{array}{l}\text { Hipotensor (Hurrell \& Puentes, } \\
\text { 2017). }\end{array}$ \\
\hline $\begin{array}{l}\text { Baccharis trimera (Less.) DC. } \\
\text { ASTERACEAE } \\
\text { Carquejilla, carqueja }\end{array}$ & $\begin{array}{l}\text { Partes aéreas frescas en } \\
\text { atados [B424] } \\
\text { Partes aéreas secas } \\
\text { fragmentadas envasadas } \\
\text { [C017] [C140] }\end{array}$ & $\begin{array}{l}\text { Hipotensor, vasodilatador (Alonso \& } \\
\text { Desmarchelier, 2005; Gómez et al., } \\
\text { 2016; Puentes, 2017). }\end{array}$ \\
\hline $\begin{array}{l}\text { Bauhinia forficata Link subsp. } \\
\text { pruinosa (Vogel) Fortunato \& } \\
\text { Wunderlin } \\
\text { FABACEAE } \\
\text { Pezuña de vaca }\end{array}$ & $\begin{array}{l}\text { Hojas secas envasadas } \\
\text { [H015] [H049] } \\
\text { Hojas secas en atado [P265] }{ }^{*}\end{array}$ & $\begin{array}{l}\text { Diurético (Alonso \& Desmarchelier, } \\
\text { 2005; Barboza et al.,2009; de } \\
\text { Souza et al., 2017). }\end{array}$ \\
\hline $\begin{array}{l}\text { Betula pendula Roth. } \\
\text { BETULACEAE } \\
\text { Abedul }\end{array}$ & $\begin{array}{l}\text { Hojas secas fragmentadas } \\
\text { envasadas [ } \mathrm{H} 071]\end{array}$ & $\begin{array}{l}\text { Diurético (Alonso \& Desmarchelier, } \\
\text { 2005). }\end{array}$ \\
\hline $\begin{array}{l}\text { Calendula officinalis } \mathrm{L} \text {. } \\
\text { ASTERACEAE } \\
\text { Caléndula }\end{array}$ & $\begin{array}{l}\text { Capitulos frescos envasados } \\
\text { [RF27] } \\
\text { Capítulos secos } \\
\text { fragmentados envasados } \\
{[\mathrm{H} 200]} \\
\text { Tintura madre }[\mathrm{H} 341]\end{array}$ & $\begin{array}{l}\text { Hipotensor (Alonso \& } \\
\text { Desmarchelier, 2005; Arora et al., } \\
\text { 2013). }\end{array}$ \\
\hline $\begin{array}{l}\text { Capsella bursa-pastoris (L.) } \\
\text { Medik. } \\
\text { BRASSICACEAE } \\
\text { Bolsa del pastor }\end{array}$ & $\begin{array}{l}\text { Partes aéreas secas } \\
\text { fragmentadas envasadas } \\
\text { [H158] }\end{array}$ & $\begin{array}{l}\text { Hipotensor, diurético (Alonso \& } \\
\text { Desmarchelier, 2005; Teixeira et al., } \\
\text { 2017). }\end{array}$ \\
\hline $\begin{array}{l}\text { Centella asiatica (L.) Urb. } \\
\text { APIACEAE } \\
\text { Centella asiática }\end{array}$ & $\begin{array}{l}\text { Partes aéreas secas } \\
\text { envasadas [H076 }]^{\star}[\mathrm{R} 176] \\
\text { Tintura madre [H321] } \\
\text { Comprimidos [H403] } \\
\text { Comprimidos (mezcla) [H404] }\end{array}$ & $\begin{array}{l}\text { Hipotensor, diurético (Arenas et al., } \\
\text { 2013; Harwoko et al., 2014; Nansy } \\
\text { et al., 2015). }\end{array}$ \\
\hline $\begin{array}{l}\text { Chamaemelum nobile (L.) All. } \\
\text { ASTERACEAE } \\
\text { Manzanilla romana }\end{array}$ & Aceite esencial [H440] & $\begin{array}{l}\text { Hipotensor, vasorelajador (Ali } \\
\text { Zeggwagh et al., 2009, 2013; Al- } \\
\text { Snafi, 2016; Mohamed et al., 2016). }\end{array}$ \\
\hline $\begin{array}{l}\text { Chrysanthemum morifolium } \\
\text { Ramat. } \\
\text { ASTERACEAE } \\
\text { Ju hua, crisantemo }\end{array}$ & $\begin{array}{l}\text { Inflorescencias secas } \\
\text { fragmentadas envasadas } \\
{[\mathrm{P} 239]^{* \star}[\mathrm{H} 420]}\end{array}$ & $\begin{array}{l}\text { Hipotensor, vasodilatador (Hurrell \& } \\
\text { Puentes, 2017; Puentes, 2017). }\end{array}$ \\
\hline $\begin{array}{l}\text { Cymbopogon citratus (DC.) Stapf } \\
\text { POACEAE } \\
\text { Pasto limón, citronella }\end{array}$ & $\begin{array}{l}\text { Bases foliares secas } \\
\text { fragmentadas a granel [H171] } \\
\text { [P240] }^{* *} \\
\text { Bases foliares frescas a } \\
\text { granel [RF08] [R022] }{ }^{* *}\end{array}$ & $\begin{array}{l}\text { Hipotensor, vasorelajante (Bastos } \\
\text { et al., 2010; Moreira et al., 2010; } \\
\text { Martins et al., 2013; García Mesa, } \\
\text { 2014). }\end{array}$ \\
\hline
\end{tabular}




\begin{tabular}{|c|c|c|}
\hline $\begin{array}{l}\text { Especies, familias, nombres } \\
\text { vulgares }\end{array}$ & Productos [Muestras] & $\begin{array}{c}\text { Actividad biológica y efectos } \\
\text { estudiados }\end{array}$ \\
\hline $\begin{array}{l}\text { Cynara cardunculus L. } \\
\text { ASTERACEAE } \\
\text { Alcachofa, alcaucil }\end{array}$ & $\begin{array}{l}\text { Partes aéreas secas } \\
\text { fragmentadas a granel [H069] } \\
\text { Té en saquitos [H093] } \\
\text { Té en saquitos (mezcla) [H411] } \\
\text { Comprimidos [H094]] } \\
\text { Comprimidos (mezcla) [H501] }\end{array}$ & $\begin{array}{l}\text { Hipotensor, diurético (Alonso \& } \\
\text { Desmarchelier, 2005; Roghani- } \\
\text { Dehkordib \& Kamkhah, 2009; } \\
\text { Arenas et al., 2013). }\end{array}$ \\
\hline $\begin{array}{l}\text { Cynodon dactylon (L.) Pers. } \\
\text { POACEAE } \\
\text { Gramilla }\end{array}$ & $\begin{array}{l}\text { Rizomas secos fragmentados } \\
\text { envasados [H248] }\end{array}$ & Hipotensor (Bharati et al., 2016). \\
\hline $\begin{array}{l}\text { Dysphania ambrosioides (L.) } \\
\text { Mosyakin \& Clemants. } \\
\text { CHENOPODIACEAE } \\
\text { Paico }\end{array}$ & $\begin{array}{l}\text { Partes aéreas secas } \\
\text { fragmentadas a granel [C002] } \\
\text { Partes aéreas secas } \\
\text { fragmentadas envasadas } \\
\text { [R128] }\end{array}$ & $\begin{array}{l}\text { Hipotensor (Alonso \& } \\
\text { Desmarchelier, 2005; Barboza et } \\
\text { al.,2009; Assaidi et al., 2014). }\end{array}$ \\
\hline $\begin{array}{l}\text { Eleutherococcus senticosus } \\
\text { (Rupr. ex Maxim.) Maxim. } \\
\text { [= Acanthopanax senticosus } \\
\text { (Rupr. \& Maxim.) Harms] } \\
\text { ARALIACEAE } \\
\text { Ginseng siberiano, eleuterococo }\end{array}$ & $\begin{array}{l}\text { Raíces secas fragmentadas } \\
\text { envasadas [P186] [R018]** } \\
\text { Cápsulas [P170] }\end{array}$ & Vasodilatador (Kwan et al., 2004). \\
\hline $\begin{array}{l}\text { Equisetum gigantum } \mathrm{L} . \\
\text { EQUISETACEAE } \\
\text { Cola de caballo }\end{array}$ & $\begin{array}{l}\text { Tallos secos fragmentados } \\
\text { envasados [H003] } \\
\text { Té en saquitos (mezcla) } \\
\text { [H410] } \\
\text { Tallos frescos en atados } \\
\text { [H090]* } \\
\text { Tallos secos fragmentados } \\
\text { envasados (mezcla) [M-71] }\end{array}$ & $\begin{array}{l}\text { Diurético (Cortella \& } \\
\text { Pochettino,1999). }\end{array}$ \\
\hline $\begin{array}{l}\text { Eschscholzia californica Cham. } \\
\text { PAPAVERACEAE } \\
\text { Amapola de California }\end{array}$ & Cápsulas [R147] & Sin datos. \\
\hline $\begin{array}{l}\text { Eucommia ulmoides Oliv. } \\
\text { EUCOMMIACEAE } \\
\text { Du zhong }\end{array}$ & $\begin{array}{l}\text { Corteza seca fragmentada } \\
\text { envasada }[\mathrm{H} 447]^{\star \star}\end{array}$ & Hipotensor (Puentes, 2017). \\
\hline $\begin{array}{l}\text { Eugenia uniflora L. } \\
\text { MYRTACEAE } \\
\text { Pitanga }\end{array}$ & $\begin{array}{l}\text { Hojas secas envasadas } \\
{[\mathrm{H} 140]}\end{array}$ & $\begin{array}{l}\text { Antihipertensivo, diurético (Consolini } \\
\text { et al., 1999, Consolini \& Sarubbio, } \\
\text { 2002; Alonso \& Desmarchelier, } \\
\text { 2005; Barboza et al., 2009; Teixeira } \\
\text { et al., 2017). }\end{array}$ \\
\hline $\begin{array}{l}\text { Foeniculum vulgare Mill. } \\
\text { APIACEAE } \\
\text { Hinojo }\end{array}$ & $\begin{array}{l}\text { Mericarpos secos a granel } \\
\text { [C047] } \\
\text { Mericarpos en polvo } \\
\text { envasados [C121] } \\
\text { Té en saquitos (mezcla) } \\
\text { [H065] } \\
\text { Tintura madre [H274] } \\
\text { Cápsulas [R159] }\end{array}$ & $\begin{array}{l}\text { Hipotensor, diurético (El Bardai et } \\
\text { al., 2001; Alonso \& Desmarchelier, } \\
\text { 2005; Badgujar et al., 2014; Texeira } \\
\text { et al., 2017). }\end{array}$ \\
\hline $\begin{array}{l}\text { Fritillaria cirrhosa D. Don } \\
\text { LILIACEAE } \\
\text { Chuan bei mu }\end{array}$ & $\begin{array}{l}\text { Bulbos secos envasados } \\
{[\mathrm{H} 455]^{\star *}}\end{array}$ & $\begin{array}{l}\text { Hipotensor, diurético (Puentes, } \\
\text { 2017). }\end{array}$ \\
\hline $\begin{array}{l}\text { Fumaria officinalis L. } \\
\text { PAPAVERACEAE } \\
\text { Fumaria }\end{array}$ & $\begin{array}{l}\text { Partes aéreas secas } \\
\text { fragmentadas envasadas } \\
{[\mathrm{H} 138]}\end{array}$ & $\begin{array}{l}\text { Hipotensor, diurético (Cortella \& } \\
\text { Pochettino, 1999; Teixeira et al., } \\
\text { 2017). }\end{array}$ \\
\hline $\begin{array}{l}\text { Gaillardia megapotamica } \\
\text { (Spreng.) Baker } \\
\text { ASTERACEAE } \\
\text { Topasaire }\end{array}$ & $\begin{array}{l}\text { Partes aéreas secas } \\
\text { fragmentadas envasadas } \\
{[\mathrm{H} 148][\mathrm{P} 153]^{*}} \\
\text { Tintura madre }[\mathrm{H} 351] \\
\end{array}$ & Sin datos. \\
\hline
\end{tabular}




\begin{tabular}{|c|c|c|}
\hline $\begin{array}{l}\text { Especies, familias, nombres } \\
\text { vulgares }\end{array}$ & Productos [Muestras] & $\begin{array}{c}\text { Actividad biológica y efectos } \\
\text { estudiados }\end{array}$ \\
\hline $\begin{array}{l}\text { Gentianella alborosea (Gilg) } \\
\text { Fabris } \\
\text { GENTIANACEAE } \\
\text { Hercampuri }\end{array}$ & $\begin{array}{l}\text { Partes aéreas secas a granel } \\
{[\text { P155 }]^{*}[\mathrm{P} 187]} \\
\text { Cápsulas }[\mathrm{P} 277]^{\star}[\mathrm{P} 377]\end{array}$ & Sin datos. \\
\hline $\begin{array}{l}\text { Handroanthus impetiginosus } \\
\text { (Mart. ex DC.) Mattos } \\
\text { BIGNONIACEAE } \\
\text { Lapacho }\end{array}$ & $\begin{array}{l}\text { Corteza seca fragmentada } \\
\text { envasada [H207] } \\
\text { Extracto líquido envasado } \\
\text { [R031] }^{* *} \\
\text { Extracto líquido envasado } \\
\text { (mezcla) [R084] } \\
\text { Tintura madre [R032] }\end{array}$ & Sin datos. \\
\hline $\begin{array}{l}\text { Harpagophytum procumbens } \\
\text { (Burch.) DC. ex Meisn. } \\
\text { PEDALIACEAE } \\
\text { Harpagofíto }\end{array}$ & $\begin{array}{l}\text { Raíces secas trozadas } \\
\text { envasadas } \\
\text { [P184] } \\
\text { Tintura madre [H294] } \\
\text { Cápsulas [R158] }\end{array}$ & $\begin{array}{l}\text { Hipotensor (Alonso \& } \\
\text { Desmarchelier, 2005; Teixeira et al., } \\
\text { 2017). }\end{array}$ \\
\hline $\begin{array}{l}\text { Heimia salicifolia (Kunth) Link } \\
\text { LYTHRACEAE } \\
\text { Quiebra arado }\end{array}$ & $\begin{array}{l}\text { Partes aéreas secas } \\
\text { fragmentadas envasadas } \\
\text { [H124] }\end{array}$ & $\begin{array}{l}\text { Vasorelajador (Barboza et al., } \\
\text { 2009). }\end{array}$ \\
\hline $\begin{array}{l}\text { Hibiscus sabdariffa L. } \\
\text { MALVACEAE } \\
\text { Rosella }\end{array}$ & $\begin{array}{l}\text { Cálices secos fragmentados y } \\
\text { envasados }[\mathrm{P} 235]^{* *}\end{array}$ & $\begin{array}{l}\text { Hipotensor, diurético (Alonso \& } \\
\text { Desmarchelier, 2005; Moradi et al., } \\
\text { 2016; Micucci et al., 2016). }\end{array}$ \\
\hline $\begin{array}{l}\text { Hieracium pilosella } \mathrm{L} \text {. } \\
\text { ASTERACEAE } \\
\text { Vellosilla }\end{array}$ & Cápsulas [H364] & Sin datos. \\
\hline $\begin{array}{l}\text { Hypericum connatum Lam. } \\
\text { HYPERICACEAE } \\
\text { Cabotoril }\end{array}$ & $\begin{array}{l}\text { Partes aéreas secas } \\
\text { fragmentadas envasadas } \\
\text { [H133] }\end{array}$ & Sin datos. \\
\hline $\begin{array}{l}\text { Jateorhiza palmata (Lam.) Miers } \\
\text { MENISPERMACEAE } \\
\text { Colombo }\end{array}$ & $\begin{array}{l}\text { Raíces secas fragmentadas } \\
\text { envasadas [H257] }\end{array}$ & Sin datos. \\
\hline $\begin{array}{l}\text { Jodina rhombifolia (Hook. \& Arn.) } \\
\text { Reissek } \\
\text { SANTALACEAE } \\
\text { Sombra de toro }\end{array}$ & $\begin{array}{l}\text { Hojas secas enteras } \\
\text { envasadas [H149] }\end{array}$ & Diurético (Teves et al., 2018). \\
\hline $\begin{array}{l}\text { Kaempferia galanga L. } \\
\text { ZINGIBERACEAE } \\
\text { Shan nai }\end{array}$ & $\begin{array}{l}\text { Rizomas secos fragmentados } \\
\text { envasados }[\mathrm{B} 033]^{* *}\end{array}$ & $\begin{array}{l}\text { Hipotensor, diurético (Puentes, } \\
\text { 2017). }\end{array}$ \\
\hline $\begin{array}{l}\text { Larrea divaricata Cav. } \\
\text { ZYGOPHYLLACEAE } \\
\text { Jarrilla }\end{array}$ & $\begin{array}{l}\text { Partes aéreas secas } \\
\text { fragmentadas envasadas } \\
{[\mathrm{H} 244][\mathrm{H} 279]^{\star *}}\end{array}$ & Sin datos. \\
\hline $\begin{array}{l}\text { Leonurus japonicus Houtt. } \\
\text { LAMIACEAE } \\
\text { Yi mu cao }\end{array}$ & $\begin{array}{l}\text { Partes aéreas secas } \\
\text { fragmentadas envasadas } \\
{[B 020]^{* \star}}\end{array}$ & $\begin{array}{l}\text { Hipotensor, vasodilatador, diurético } \\
\text { (Puentes, 2017). }\end{array}$ \\
\hline $\begin{array}{l}\text { Lepidium didymum L. } \\
\text { BRASSICACEAE } \\
\text { Quimpe }\end{array}$ & $\begin{array}{l}\text { Partes aéreas secas } \\
\text { fragmentadas envasadas } \\
\text { (mezcla) [M-73] } \\
\text { Partes aéreas secas } \\
\text { fragmentadas envasadas } \\
\text { [H188] }\end{array}$ & Sin datos. \\
\hline $\begin{array}{l}\text { Ligaria cuneifolia (Ruiz \& Pav.) } \\
\text { Tiegh. } \\
\text { LORANTHACEAE } \\
\text { Muérdago criollo/Liga }\end{array}$ & $\begin{array}{l}\text { Partes aéreas secas } \\
\text { fragmentadas envasadas } \\
\text { [H193] } \\
\text { Partes aéreas } \\
\text { secas fragmentadas } \\
\text { envasadas[M-72] }\end{array}$ & $\begin{array}{l}\text { Hipotensor (Cortella \& } \\
\text { Pochettino,1999). }\end{array}$ \\
\hline
\end{tabular}




\begin{tabular}{|c|c|c|}
\hline $\begin{array}{l}\text { Especies, familias, nombres } \\
\text { vulgares }\end{array}$ & Productos [Muestras] & $\begin{array}{l}\text { Actividad biológica y efectos } \\
\text { estudiados }\end{array}$ \\
\hline $\begin{array}{l}\text { Ligusticum striatum DC. } \\
\text { APIACEAE } \\
\text { Chuan xiong }\end{array}$ & $\begin{array}{l}\text { Rizomas secos fragmentados } \\
\text { envasados [B027] }]^{* \star}\end{array}$ & Hipotensor (Puentes, 2017). \\
\hline $\begin{array}{l}\text { Lonicera japonica Thunb. } \\
\text { CAPRIFOLIACEAE } \\
\text { Jin yin hua, madreselva }\end{array}$ & $\begin{array}{l}\text { Botones y flores secos } \\
\text { fragmentados envasados } \\
{[\text { [B017] }]^{\star *}}\end{array}$ & Hipotensor (Puentes, 2017). \\
\hline $\begin{array}{l}\text { Lycium barbarum L. } \\
\text { SOLANACEAE } \\
\text { Goji }\end{array}$ & $\begin{array}{l}\text { Frutos secos a granel }[\mathrm{H} 037] \\
\text { Frutos secos envasados } \\
{\left[\mathrm{RF}^{2}\right]^{\star *}[\mathrm{D} 001]}\end{array}$ & Hipotensor (Zhang et al., 2015). \\
\hline $\begin{array}{l}\text { Matricaria chamomilla L. } \\
\text { (= M. recutita L.) } \\
\text { ASTERACEAE } \\
\text { Manzanilla }\end{array}$ & $\begin{array}{l}\text { Plantas frescas a granel } \\
{[\text { B427]* }} \\
\text { Partes aéreas secas } \\
\text { fragmentadas envasadas } \\
\text { [C008][H089] } \\
\text { Tintura madre [H357] } \\
\text { Té en saquitos [H361] [H016] } \\
\text { Té en saquitos (mezcla) } \\
\text { [H047] }\end{array}$ & $\begin{array}{l}\text { Hipotensor, diurético (Alonso \& } \\
\text { Desmarchelier; Moradi et al., 2016). }\end{array}$ \\
\hline $\begin{array}{l}\text { Maytenus officinalis Mabb. } \\
\text { CELASTRACEAE } \\
\text { Congorosa }\end{array}$ & $\begin{array}{l}\text { Hojas y ramitas secas } \\
\text { fragmentadas envasadas } \\
{[\mathrm{H} 139]}\end{array}$ & Sin datos. \\
\hline $\begin{array}{l}\text { Minthostachys verticillata } \\
\text { (Griseb.) Epling } \\
\text { LAMIACEAE } \\
\text { Peperina }\end{array}$ & $\begin{array}{l}\text { Partes aéreas secas } \\
\text { fragmentadas envasadas } \\
\text { [C021] }\end{array}$ & Sin datos. \\
\hline $\begin{array}{l}\text { Morinda citrifolia L. } \\
\text { RUBIACEAE } \\
\text { Noni }\end{array}$ & $\begin{array}{l}\text { Frutos secos pulverizados } \\
\text { envasados [H161] } \\
\text { Cápsulas [H379] } \\
\text { Extracto líquido [PN03] }\end{array}$ & $\begin{array}{l}\text { Hipotensor, diurético (Alonso \& } \\
\text { Desmarchelier, 2005; Puentes \& } \\
\text { Hurrell, 2015; Cristina, 2016; Wigati } \\
\text { et al., 2017). }\end{array}$ \\
\hline $\begin{array}{l}\text { Nelumbo nucifera Gaertn. } \\
\text { NELUMBONACEAE } \\
\text { Lian, loto sagrado }\end{array}$ & $\begin{array}{l}\text { Semillas secas envasadas } \\
\text { [BH20] }^{* *} \\
\text { Pasta de semillas envasada } \\
\text { [R028] }^{* *} \\
\text { Plúmulas secas enteras } \\
\text { envasadas [B018 }]^{* *}\end{array}$ & Hipotensor (Puentes, 2017). \\
\hline $\begin{array}{l}\text { Oenothera biennis L. } \\
\text { ONAGRACEAE } \\
\text { Onagra }\end{array}$ & $\begin{array}{l}\text { Aceite en perlas envasado } \\
\text { [R162] }\end{array}$ & $\begin{array}{l}\text { Hipotensor (Alonso \& } \\
\text { Desmarchelier, 2005). }\end{array}$ \\
\hline $\begin{array}{l}\text { Olea europea L. } \\
\text { OLEACEAE } \\
\text { Olivo }\end{array}$ & $\begin{array}{l}\text { Hojas secas envasadas } \\
{[\mathrm{H} 191]}\end{array}$ & $\begin{array}{l}\text { Hipotensor (Cortella \& } \\
\text { Pochettino,1999; Alonso \& } \\
\text { Desmarchelier, 2005; Moradi et al., } \\
\text { 2016; Micucci et al., 2016; Teixeira } \\
\text { et al., 2017). }\end{array}$ \\
\hline $\begin{array}{l}\text { Orthosiphon stamineus Benth. } \\
\text { LAMIACEAE } \\
\text { Ortosifón }\end{array}$ & $\begin{array}{l}\text { Partes aéreas secas } \\
\text { fragmentadas envasadas } \\
\text { [H429] } \\
\text { Cápsulas [SD38] }\end{array}$ & $\begin{array}{l}\text { Hipotensor, , diurético (Alonso \& } \\
\text { Desmarchelier, 2005; Arenas et al., } \\
\text { 2013; Ashraf et al., 2018). }\end{array}$ \\
\hline $\begin{array}{l}\text { Passiflora caerulea L. } \\
\text { PASSIFLORACEAE } \\
\text { Pasionaria }\end{array}$ & $\begin{array}{l}\text { Partes aéreas secas } \\
\text { envasadas [H081] } \\
\text { Té en saquitos (mezcla) } \\
\text { [H410] }\end{array}$ & Sin datos. \\
\hline $\begin{array}{l}\text { Passiflora edulis Sims f. } \\
\text { flavicarpa O. Deg. } \\
\text { PASSIFLORACEAE } \\
\text { Maracuyá }\end{array}$ & $\begin{array}{l}\text { Partes aéreas secas } \\
\text { envasadas [H108] } \\
\text { Té en saquitos (mezcla) } \\
\text { [H410] }\end{array}$ & $\begin{array}{l}\text { Hipotensor (Ichimura et al., 2006; } \\
\text { Lewis et al., 2013; Konta et al., } \\
\text { 2014). }\end{array}$ \\
\hline
\end{tabular}




\begin{tabular}{|c|c|c|}
\hline $\begin{array}{l}\text { Especies, familias, nombres } \\
\text { vulgares }\end{array}$ & Productos [Muestras] & $\begin{array}{c}\text { Actividad biológica y efectos } \\
\text { estudiados }\end{array}$ \\
\hline $\begin{array}{l}\text { Phyllanthus niruri L. } \\
\text { EUPHORBIACEAE } \\
\text { Chancapiedra }\end{array}$ & $\begin{array}{l}\text { Partes aéreas secas } \\
\text { envasadas [P205] [P195]* } \\
\text { Cápsulas [H441]* }\end{array}$ & $\begin{array}{l}\text { Hipotensor, diurético (Barboza et } \\
\text { al.,2009; Filho, 2018). }\end{array}$ \\
\hline $\begin{array}{l}\text { Phyllanthus sellowianus } \\
\text { (Klotzsch) Müll. Arg. } \\
\text { EUPHORBIACEAE } \\
\text { Sarandí blanco }\end{array}$ & $\begin{array}{l}\text { Corteza seca fragmentada } \\
\text { envasada [H067] }\end{array}$ & $\begin{array}{l}\text { Diurético (Alonso \& Desmarchelier, } \\
\text { 2005; Barboza et al., 2009). }\end{array}$ \\
\hline $\begin{array}{l}\text { Pilocarpus pennatifolius Lem. } \\
\text { RUTACEAE } \\
\text { Jaborandi }\end{array}$ & $\begin{array}{l}\text { Partes aéreas secas } \\
\text { fragmentadas envasadas } \\
\text { [H169] }\end{array}$ & Sin datos. \\
\hline $\begin{array}{l}\text { Plantago ovata Forssk. } \\
\text { PLANTAGINACEAE } \\
\text { Psyllium }\end{array}$ & $\begin{array}{l}\text { Semillas secas a granel } \\
{\left[^{H} 393\right]^{* *}[R 402]} \\
\text { Material pulverizado } \\
\text { envasado }[\mathrm{H} 325]\end{array}$ & Hipotensor (Solá et al., 2010). \\
\hline $\begin{array}{l}\text { Polygonum aviculare L. } \\
\text { POLYGONACEAE } \\
\text { Sanguinaria }\end{array}$ & $\begin{array}{l}\text { Partes aéreas secas } \\
\text { fragmentadas envasadas } \\
\text { [H197] } \\
\text { Partes aéreas secas } \\
\text { fragmentadas envasadas } \\
\text { (mezcla) [M-72] }\end{array}$ & $\begin{array}{l}\text { Diurético, vasodilatador (Cortella \& } \\
\text { Pochettino,1999; Yin et al., 2005) }\end{array}$ \\
\hline $\begin{array}{l}\text { Prosopis strombulifera (Lam.) } \\
\text { Benth. } \\
\text { FABACEAE } \\
\text { Mastuerzo }\end{array}$ & $\begin{array}{l}\text { Frutos secos envasados } \\
{[\mathrm{H} 022][\mathrm{H} 088]^{*}}\end{array}$ & Sin datos. \\
\hline $\begin{array}{l}\text { Prunella vulgaris } \mathrm{L} \text {. } \\
\text { LAMIACEAE } \\
\text { Xia ku cao }\end{array}$ & $\begin{array}{l}\text { Inflorescencias secas } \\
\text { fragmentadas envasadas } \\
{[\text { B025] }]^{* *}}\end{array}$ & Hipotensor (Puentes, 2017). \\
\hline $\begin{array}{l}\text { Psidium guajava L. } \\
\text { MYRTACEAE } \\
\text { Arazá }\end{array}$ & $\begin{array}{l}\text { Hojas secas fragmentadas } \\
\text { envasadas [H260] }\end{array}$ & Hipotensor (Ojewole, 2005). \\
\hline $\begin{array}{l}\text { Salvia hispanica L. } \\
\text { LAMIACEAE } \\
\text { Chía }\end{array}$ & $\begin{array}{l}\text { Semillas secas envasadas } \\
\text { [H042] } \\
\text { Semillas secas tostadas } \\
\text { envasadas [H113] } \\
\text { Harina envasada [H107] } \\
\text { Harina a granel [H313] } \\
\text { Comprimidos [R170] } \\
\text { Aceite en botella [R057] }\end{array}$ & $\begin{array}{l}\text { Hipotensor (Ulbricht et al., 2009; } \\
\text { Ullah et al., 2016). }\end{array}$ \\
\hline $\begin{array}{l}\text { Schisandra chinensis (Turcz.) } \\
\text { Baill. } \\
\text { SCHISANDRACEAE } \\
\text { Wu wei zi, eschisandra }\end{array}$ & $\begin{array}{l}\text { Frutos secos envasados } \\
\text { [P208] [RF59] } \\
\text { Cápsulas (mezcla) [H323] }\end{array}$ & $\begin{array}{l}\text { Hipotensor (Kim et al., 2015; } \\
\text { Cristina, 2016). }\end{array}$ \\
\hline $\begin{array}{l}\text { Senecio nutans Sch. Bip. } \\
\text { ASTERACEAE } \\
\text { Chachacoma, chachacoma de la } \\
\text { Puna }\end{array}$ & $\begin{array}{l}\text { Partes aéreas secas } \\
\text { fragmentadas envasadas } \\
{[\mathrm{H} 234][\mathrm{P} 154]}\end{array}$ & $\begin{array}{l}\text { Hipotensor (Alonso \& Desmarchilier, } \\
\text { 2005; Barboza et al., 2009). }\end{array}$ \\
\hline $\begin{array}{l}\text { Smallanthus sonchifolius } \\
\text { (Poepp.) H. Rob. } \\
\text { ASTERACEAE } \\
\text { Yacón }\end{array}$ & $\begin{array}{l}\text { Hojas secas envasadas } \\
{[\mathrm{H} 332]^{\star *}[\mathrm{R} 177]} \\
\text { Tintura madre }[\mathrm{H} 285] \\
\text { Cápsulas }[\mathrm{H} 286]^{*}[\mathrm{H} 293]^{*} \\
\text { Extracto líquido }[\mathrm{P} 275]^{*}\end{array}$ & Sin datos. \\
\hline $\begin{array}{l}\text { Solanum sisymbriifolium Lam. } \\
\text { SOLANACEAE } \\
\text { Espina colorada }\end{array}$ & $\begin{array}{l}\text { Raíces secas fragmentadas } \\
\text { envasadas }[\mathrm{H} 126]\end{array}$ & $\begin{array}{l}\text { Hipotensor (Barboza et al., 2009; } \\
\text { lbarrola et al., 2000, 2011). }\end{array}$ \\
\hline
\end{tabular}




\begin{tabular}{|c|c|c|}
\hline $\begin{array}{l}\text { Especies, familias, nombres } \\
\text { vulgares }\end{array}$ & Productos [Muestras] & $\begin{array}{c}\text { Actividad biológica y efectos } \\
\text { estudiados }\end{array}$ \\
\hline $\begin{array}{l}\text { Stevia rebaudiana (Bertoni) } \\
\text { Bertoni } \\
\text { ASTERACEAE } \\
\text { Estevia, yerba dulce }\end{array}$ & $\begin{array}{l}\text { Plantas frescas [B415] } \\
\text { Hojas secas envasadas } \\
\text { [H198] [P147] [M006] }^{*} \\
\text { Tintura madre [H350] } \\
\text { Extracto líquido [P322] } \\
\text { Extracto en polvo [P321] }\end{array}$ & $\begin{array}{l}\text { Hipotensor, diurético (Melis, 1995; } \\
\text { Alonso \& Desmarchilier, 2005; } \\
\text { Momtazi-Borojeni et al., 2017; } \\
\text { Texeira et al., 2017). }\end{array}$ \\
\hline $\begin{array}{l}\text { Tagetes minuta L. } \\
\text { ASTERACEAE } \\
\text { Suico, huacatay }\end{array}$ & $\begin{array}{l}\text { Plantas frescas [B403] } \\
\text { Partes aéreas secas } \\
\text { envasadas [H415] }\end{array}$ & $\begin{array}{l}\text { Hipotensor (Barboza et al., 2009; } \\
\text { Ranilla et al., 2010). }\end{array}$ \\
\hline $\begin{array}{l}\text { Tanacetum parthenium (L.) Sch. } \\
\text { Bip. } \\
\text { ASTERACEAE } \\
\text { Santa María }\end{array}$ & $\begin{array}{l}\text { Partes aéreas secas } \\
\text { fragmentadas envasadas } \\
{[\mathrm{H} 223]}\end{array}$ & Sin datos. \\
\hline $\begin{array}{l}\text { Tanacetum vulgare } \mathrm{L} . \\
\text { ASTERACEAE } \\
\text { Tanaceto }\end{array}$ & $\begin{array}{l}\text { Partes aéreas secas } \\
\text { fragmentadas envasadas } \\
{[\mathrm{H} 214]}\end{array}$ & Hipotensor (Lahlou et al., 2008). \\
\hline $\begin{array}{l}\text { Taraxacum officinale Weber ex F. } \\
\text { H. Wigg. } \\
\text { ASTERACEAE } \\
\text { Diente de león }\end{array}$ & $\begin{array}{l}\text { Partes aéreas secas a granel } \\
{[\mathrm{C} 087][\mathrm{H} 100]^{*}} \\
\text { Tintura madre }[\mathrm{H} 337]\end{array}$ & $\begin{array}{l}\text { Hipotensor, diurético (Alonso \& } \\
\text { Desmarchilier, 2005; Veiga et al., } \\
\text { 2005; Teixeira et al., 2017). }\end{array}$ \\
\hline $\begin{array}{l}\text { Tilia cordata Mill. } \\
\text { TILIACEAE } \\
\text { Tilo }\end{array}$ & $\begin{array}{l}\text { Partes aéreas secas } \\
\text { envasadas [H205] } \\
\text { Té en saquitos [H043] }\end{array}$ & $\begin{array}{l}\text { Diurético (Alonso \& Desmarchilier, } \\
\text { 2005). }\end{array}$ \\
\hline $\begin{array}{l}\text { Urtica urens L. } \\
\text { URTICACEAE } \\
\text { Ortiga }\end{array}$ & $\begin{array}{l}\text { Partes aéreas secas } \\
\text { envasadas [H196] }\end{array}$ & $\begin{array}{l}\text { Diurético, hipotensor (Cortella \& } \\
\text { Pochettino,1999). }\end{array}$ \\
\hline $\begin{array}{l}\text { Vachellia aroma (Gillies ex Hook. } \\
\text { \& Arn.) Seigler \& Ebinger } \\
\text { FABACEAE } \\
\text { Tusca }\end{array}$ & $\begin{array}{l}\text { Corteza seca fragmentada } \\
\text { envasada [H075] }\end{array}$ & Sin datos. \\
\hline $\begin{array}{l}\text { Vachellia caven (Molina) Seigler \& } \\
\text { Ebinger } \\
\text { FABACEAE } \\
\text { Espinillo }\end{array}$ & $\begin{array}{l}\text { Hojas y flores secas } \\
\text { fragmentadas envasadas } \\
\text { [H085] }\end{array}$ & Sin datos. \\
\hline $\begin{array}{l}\text { Valeriana officinalis } \mathrm{L} \text {. } \\
\text { VALERANIACEAE } \\
\text { Valeriana }\end{array}$ & $\begin{array}{l}\text { Raíces secas fragmentadas } \\
\text { envasadas }[\mathrm{H} 078]^{*}[\mathrm{R} 401] \\
\text { Comprimidos }[\mathrm{H} 326] \\
\text { Tintura madre [D106] }\end{array}$ & $\begin{array}{l}\text { Hipotensor (Alonso \& Desmarchilier, } \\
\text { 2005; Teixeira et al., 2017). }\end{array}$ \\
\hline $\begin{array}{l}\text { Zea mays } \mathrm{L} \text {. } \\
\text { POACEAE } \\
\text { Estigmas de maíz, barba de choclo }\end{array}$ & $\begin{array}{l}\text { Estigmas secos a granel } \\
\text { [H163] }^{*} \\
\text { Estigmas secos envasados }^{[R 097]}\end{array}$ & $\begin{array}{l}\text { Hipotensor, diurético (Alonso \& } \\
\text { Desmarchilier, 2005; George \& Idu, } \\
\text { 2015). }\end{array}$ \\
\hline $\begin{array}{l}\text { Zingiber officinale Roscoe } \\
\text { ZINGIBERACEAE } \\
\text { Jengibre }\end{array}$ & $\begin{array}{l}\text { Rizomas secos fragmentados } \\
\text { a granel [H453] } \\
\text { Rizomas secos pulverizados } \\
\text { a granel [H454] } \\
\text { Cápsulas (mezcla) [P170] }\end{array}$ & Hipotensor (Akinyemi et al., 2013). \\
\hline
\end{tabular}

Del total de especies presentadas, 20 no presentan trabajos académicos que se correlacionen con el uso local como hipotensor difundido en el AMBA.

En el Mercado Boliviano se registraron productos pertenecientes a 16 especies: 7 tienen todos sus productos tanto en ese mercado como en el circuito comercial general. Por otro lado, 16 especies presentan productos en el Barrio Chino de Belgrano: 6 tienen todos sus productos tanto en el Barrio chino como en el circuito comercial general (visibles): 
Las 10 restantes poseen todos sus productos exclusivamente en el Barrio Chino (invisibles). Asimismo, se destaca la comercialización de plantas frescas de Artemisia absinthium, Baccharis trimera, Matricaria chamomilla, Stevia rebaudiana y Tagetes minuta, que se emplean con fines medicinales.

\section{Discusión y Conclusiones}

De las 83 especies relevadas, 10 (12\%) presentan todos sus productos en el Barrio Chino por lo tanto son invisibles para la mayoría de la población urbana local, mientras que las 73 $(88 \%)$ restantes son visibles. En el Barrio Chino se comercializan plantas medicinales que aún no ingresan al circuito comercial general, pero es posible que estas especies invisibles se tornen visibles en un futuro próximo como sucedió con Astragalus mongholicus, Lycium barbarum y Schisandra chinensis, cuyos productos se han registrado en distintas dietéticas y herboristerías del área del estudio (Hurrell \& Puentes, 2017; Puentes, 2017). Estas especies son ejemplos de cómo el proceso de visualización evidencia la dinámica del conocimiento botánico urbano y el rol de las dietéticas como agentes de visualización (Arenas et al., 2011; Cristina, 2016). Las especies exclusivas del Barrio Chino identificadas como hipotensoras, representan el componente ligado a las tradiciones del CB de los grupos de inmigrantes (Hurrell \& Pochettino, 2014). Con respecto al Mercado Boliviano, si bien todas las especies son visibles, algunos de sus productos son exclusivos de este segmento de inmigrantes. Las plantas medicinales que se expenden en forma fresca son cultivadas en la región rioplatense, lo cual aumenta la agrodiversidad local (Puentes \& Hurrell, 2015). Los resultados obtenidos respecto a la exclusividad de los productos del Mercado Boliviano ponen de relevancia el rol y la importancia que tienen los sectores de inmigrantes urbanos en el aumento de la diversidad de productos y sus saberes asociados, es decir, la diversidad biocultural en la región (Hurrell, 2014; Puentes, 2016).

Las especies que son visibles, amplían las posibilidades de elección de los consumidores locales al momento de buscar alternativas para el tratamiento de la hipertensión. Es importante destacar que, dentro de estas 73 especies visibles, 20 no poseen estudios académicos que se correlacionen con el uso local hipotensor. Esta información puede ser utilizada para alentar a realizar nuevas investigaciones que se correlacionen con dicho uso. Entre las especies que presentan mayor cantidad de estudios académicos vinculados al tratamiento de la hipertensión se pueden mencionar: Allium sativum, "ajo"; Annona muricata, "graviola"; Foeniculum vulgare, "hinojo"; Olea europea, "olivo" y Stevia rebaudiana, "yerba dulce o estevia"; esta última presenta la mayor variedad de productos debido en gran medida, a su empleo como antidiabética (Puentes, 2016). Hasta la fecha, ciertas especies como Artemisia absinthium, Betula pendula, Eleutherococcus senticosus, Jodina rhombifolia, Phyllanthus sellowianus no poseen estudios que evalúen $\mathrm{su}$ efecto hipotensor, pero presentan efectos vinculados (diurético, vasodilatador), que pueden explicar su difusión como plantas hipotensoras.

En comparación con estudios previos, las plantas antidiabéticas concentran la mayor cantidad de especies (115), seguidas por las hipotensoras (83), hipocolesterolémicas (82), adelgazantes (60) y potenciadores cognitivos (30) (Arenas et al., 2011, 2015; Hurrell et al., 2015a, b; Puentes, 2016). La hipertensión, como la diabetes, hipercolesterolemia, obesidad, la depresión y el estrés, son enfermedades frecuentes en los contextos pluriculturales urbanos, ligadas al ritmo de vida en las grandes ciudades, según se desprende de los trabajos realizados.

Esta y otras contribuciones similares aportan datos etnobotánicos sobre plantas vinculadas al tratamiento de la hipertensión y la validez científica de su uso, información que podría ser utilizada por los consumidores que deciden emplear esas plantas. Asimismo, si bien la validez científica es útil al momento de elegir un producto medicinal, cabe destacar que es de suma importancia tener responsabilidad en el uso adecuado de esta información para evitar el consumo de dosis inadecuadas perjudiciales para la salud. Desde la Etnobotánica urbana, se contribuye al estudio del conocimiento botánico local a través del análisis de su composición y su dinámica; reflejado en el registro de los usos de las especies y la circulación de sus productos. 


\section{Agradecimientos}

El autor agradece a la Dra. Patricia M. Arenas y el Dr. Julio A. Hurrell por la lectura crítica del manuscrito y sus oportunas sugerencias. A la Dra. María Lelia Pochettino y a los integrantes del LEBA por su inestimable ayuda, a la Méd. Cecilia I. Schweitzer por sus aportes en torno a los aspectos médicos de la hipertensión y a todos los informantes que participaron de los trabajos de campo. La línea de investigación es llevada adelante con el aporte financiero de la Universidad Nacional de La Plata y del Consejo Nacional de Investigaciones Científicas y Técnicas (CONICET) Argentina.

\section{Bibliografía}

ADEFEGHA, S. A., S. I. OYELEYE \& G. OBOH. 2015. Distribution of Phenolic Contents, Antidiabetic Potentials, Antihypertensive Properties, and Antioxidative Effects of Soursop (Annona muricata L.) Fruit Parts In Vitro. Biochemistry Research International 2015: 347673. https://doi.org/10.1155/2015/347673

AKINYEMI, A. J., A. O., ADEMILUYI \& G. OBOH. 2013. Aqueous extracts of two varieties of ginger (Zingiber officinale) inhibit angiotensin I-converting enzyme, iron (II), and sodium nitroprusside-induced lipid peroxidation in the rat heart in vitro. Journal of Medicine Food 16: 641-646. ttps://doi.org/10.1089/jmf.2012.0022

ALBUQUERQUE, U. P., L. V. F. CRUZ DA CUNHA, R. F. P. LUCENA \& R. R. N. ALVES (Eds.). 2014. Methods and techniques in Ethnobiology and Ethnoecology. 480 pp. Springer-Humana Press, New York. https://doi.org/10.1007/978-1-4614-8636-7

ALI, S. I., B. GOPALAKRISHNAN \& V. VENKATESALU. 2017. Pharmacognosy, Phytochemistry and Pharmacological Properties of Achillea millefolium L.: A Review. Phytotherapy Research. 31: 1140-1161. https://doi.org/10.1002/ptr.5840

ALI ZEGGWAGH, N., A. MOUFID, J. B. MICHEL \& M. EDDOUKS. 2009. HypotensiveEffect of Chamaemelum nobile Aqueous Extract in Spontaneously Hypertensive Rats. Clinical and Experimental Hypertension 31: 440450. https://doi.org/10.1080/10641960902825453

ALI ZEGGWAGH, N., J. B. MICHEL \& M. EDDOUKS. 2013. Vascular Effects of Aqueous Extract of Chamaemelum nobile: In Vitro Pharmacological Studies in Rats. Clinical and Experimental Hypertension 35: 200-206. https://doi.org/10.3109/10641963.2012.712179
ALONSO, J. \& C. DESMARCHELIER. 2005. Plantas medicinales autóctonas de la Argentina. 663 pp. Ed. Lola, Buenos Aires.

AL-SNAFI, A. E. 2016. Medical importance of Anthemis nobilis (Chamaemelum nobile) - a review. Asian Journal of Pharmaceutical Science \& Technology 6: 89-95.

ARENAS, P. M., I. CRISTINA, J. P. PUENTES., F. BUET COSTANTINO, J. A. HURRELL \& M. L. POCHETTINO. 2011. Adaptógenos: plantas medicinales tradicionales comercializadas como suplementos dietéticos en la conurbación. Buenos Aires-La Plata (Argentina). Bonplandia 20: 251 264.

ARENAS, P. M., S. MOLARES, A. AGUILAR CONTRERAS, B. DOUMECQ \& F. GABRIELLI. 2013. Ethnobotanical, micrographic and pharmacological features of plant-based weightloss products sold in naturist stores in Mexico City: the need for better quality control Acta Botanica Brasilica 27: 560-579. https://doi.org/10.1590/S0102-33062013000300014 ARENAS, P. M., B. DOUMECQ, J. P. PUENTES \& J. A. HURRELL. 2015. Algas y plantas comercializadas como adelgazantes en el Área Metropolitana de Buenos Aires, Argentina. Gaia Scientia 9: 32-40.

ARORA, D., A. RANI \& A. SHARMA. 2013. A review on phytochemistry and ethnopharmacological aspects of genus Calendula. Pharmacognosy Reviews 7: 179-187. https://doi.org/10.4103/0973-7847.120520

ASHRAF, K., S. SULTAN \& A. ADAM. 2018. Orthosiphon stamineus Benth. is an Outstanding Food Medicine: Review of Phytochemical and Pharmacological Activities. Journal of Pharmacy \& Bioallied Sciences 10: 109-118. https://doi.org/10.4103/JPBS.JPBS 253 17

ASSAIDI, A., A. LEGSSYER, A. BERRICHI, M. AZIZ, H. MEKHFI, M. BNOUHAM \& A. ZIYYAT. 2014. Hypotensive property of Chenopodium ambrosioides in anesthetized normotensive rats. Journal of Complementary and Integrative Medicine 11: 1-7. https://doi.org/10.1515/jcim-2013-0045

BADGUJAR, S. B., V. V. PATEL \& A. H. BANDIVDEKAR. 2014. Foeniculum vulgare Mill.: A review of its botany, phytochemistry, pharmacology, contemporary application, and toxicology. BioMed Research International 1-32. https://doi.org/10.1155/2014/842674

BARBOZA, G., J. CANTERO, C. O. NUÑEZ, A. PACCIARONI \& L. ARIZA ESPINAR. 2009. Medicinal plants: a general review and a phytochemical and ethnopharmacological screening of the native Argentine Flora. Kurtziana 34: 7-365.

BASTOS, J. F. A., I. J. A. MOREIRA, T. P. RIBEIRO, I. A. MEDEIROS, A. R. ANTONIOLLI, D. P. DE SOUSA \& M. R. V. SANTOS. 2010. Hypotensive and vasorelaxant effects of citronellol, a monoterpene 
alcohol, in rats. Basic \& Clinical Pharmacology \& Toxicology 106: 331-337.

https://doi.org/10.1111/j.1742-7843.2009.00492.x

BHANDARI, P. R. 2012. Garlic (Allium sativum L.): A review of potential therapeutic applications. International Journal of Green Pharmacy 6: 118-129. https://doi.org/10.4103/0973-8258.102826

BHARATI, D., S. RAWAT, P. SHARMA \& B. SHRIVASTAVA. 2016. Evaluation of in vivo efficacy of aqueous extract of aerial parts of Cynodon dactylon in rats with simultaneous type 2 diabetes and hypertension. Current Bioactive Compounds 12: 25-33.

https://doi.org/10.2174/157340721266616021023 0059

BRANKOVIC, S., M. RADENKOVIC, D. KITIC, S. VELJKOVIC, V. IVETIC, D. PAVLOVIC \& B. MILADINOVIC. 2011. Comparison of the hypotensive and bradycardic activity of ginkgo, garlic, and onion extracts. Clinical and Experimental Hypertension 33: 95-99. https://doi.org/10.3109/10641963.2010.531833

BRÄNDLE, M. S., A. L. MAKDESSI, R. K. WEBER, K. DIETZ \& R. JACOB. 1997. Prolongation of life span in hypertensive rats by dietary interventions. Effects of garlic and linseed oil. Basic Research in Cardiolology 92: 223-232. https://doi.org/10.1007/BF00788517

CHAUPIS-MEZA, D., J. ROJAS, M. GASCO \& G. F. GONZALES. 2014. Hypotensive effect of extract of macerated garlic (Allium sativum) for 18 weeks in an in vivo experimental model. Revista Peruana de Medicina Experimental y Salud Pública 31: 461-466. https://doi.org/10.17843/ rpmesp.2014.313.81

CONSOLINI, A. E., O. A. BALDINI \& A. G. AMAT. 1999. Pharmacological basis for the empirical use of Eugenia uniflora L. (Myrtaceae) as antihypertensive. Journal of Ethnopharmacology 66: 33-39. https://doi.org/10.1016/S0378-8741(98)00194-9

CONSOlini, A. E. \& M. G. SARUBBIO.2002. Pharmacological effects of Eugenia uniflora (Myrtaceae) aqueous crude extract on rat's heart. Journal of Ethnopharmacology 81: 57-63. https://doi.org/10.1016/S0378-8741(02)00039-9

CORIA-TÉLLEZ, A. V., E. MONTALVO-GÓNZALEZ, E. M. YAHIA \& E. N. OBLEDO-VÁZQUEZ. 2018. Annona muricata: A comprehensive review on its traditional medicinal uses, phytochemicals, pharmacological activities, mechanisms of action and toxicity. Arabian Journal of Chemistry 11: 662691. https://doi.org/10.1016/j.arabjc.2016.01.004

CORTEllA, A. R \& M. L. POCHETTINO.1999. Plants employed for hypotensive infusions in urban areas of Argentina. Pharmaceutical Biology 37: 97-104. https://doi.org/10.1076/phbi.37.2.97.6085
CRISTINA, I. 2016. Caracterización botánica y etnobotánica de las plantas empleadas como adaptógenos en algunas áreas urbanas de Argentina. Tesis doctoral, Universidad Nacional de La Plata, Argentina. $230 \mathrm{pp}$.

CUASSOLO, F., A. H. LADIO \& C. EZCURRA. 2010. Aspectos de la comercialización y control de calidad de las plantas medicinales más vendidas en una comunidad urbana del NO de la Patagonia Argentina. Boletín Latinoamericano y del Caribe de Plantas Medicinales y Aromáticas 9: 166-176.

DE SOUZA, P, A. Jr. GASPAROTTO, S. CRESTANI, M. É. STEFANELlO, M. C. MARQUES, J. E. DA SILVA-SANTOS \& C. A. KASSUYA. 2011. Hypotensive mechanism of the extracts and artemetin isolated from Achillea millefolium L. (Asteraceae) in rats. Phytomedicine 18: 819-825. https://doi.org/10.1016/j.phymed.2011.02.005

DE SOUZA, P, S. CRESTANI, C. DA SILVA RDE, F. GASPAROTTO, C. A. KASSUYA, J. E. DA SILVA-SANTOS \& A. JUNIOR GASPAROTTO. 2013. Involvement of bradykinin and prostaglandins in the diuretic effects of Achillea millefolium L. (Asteraceae). Journal of Ethnopharmacology 149: 157-161. https://doi.org/10.1016/j.jep.2013.06.015

DE SOUZA, P, L. M. DA SILVA, T. BOEING, L. B. SOMENSI, C. C. CECHINEL-ZANCHETT, A. CAMPOS, C. M. A. KRUEGER, J. K. BASTOS, V. CECHINEL-FILHO \& S. F. ANDRADE. 2017. Influence of prostanoids in the diuretic and matriuretic effects of extracts and kaempferitrin from Bauhinia forficata Link leaves in rats. Phytotherapy Research 31: 1521-1528.

EL BARDAI, S., B. LYOUSSI, M. WIBO \& N. MOREL. 2001. Pharmacological evidence of hypotensive activity of Marrubium vulgare and Foeniculum vulgare in spontaneously hypertensive rat. Clinical and Experimental Hypertension 23: 329-343. https://doi.org/10.1081/CEH-100102671

ETKIN, N. L. \& T. TICKTIN. 2010. Advancing an ethnoecological perspective that integrates theory and method in ethnobotany. En ALBUQUERQUE U. P. \& N. HANAZAKI (eds.), Recent developments and case studies in Ethnobotany, pp. 33-57. SBEE/ NUPEEA, Recife, Brasil.

FILHO, V. C. 2018. Phyllanthus niruri L. En ALBUQUERQUE, U. P., U. PATIL \& A. MÁTHÉ (eds.), Medicinal and aromatic plantas of South America, pp. 367-371. Springer, Dordrecht, Netherlands. https://doi.org/10.1007/978-94-024-1552-0 33

DE SOUZA, P., L. M. DA SILVA, T. BOEING, L. B. SOMENSI, C. C. CECHINEL-ZANCHETT, A. CAMPOS, C. M. A. KRUEGER, J. K. BASTOS, V. CECHINEL-FILHO \& S. F. ANDRADE. 2017. Influence of prostanoids in the diuretic and 
natriuretic effects of extracts and kaempferitrin from Bauhinia forficata Link leaves in rats. Phytotherapy Research 31: 1521-1528. Epub 2017 Jul 28. https://doi.org/10.1002/ptr.5876

FORSTALL, R. L., R. P. GREENE \& J. B. PICK. 2004. Which are the largest? City Futures Conference, Chicago, USA.

GARCÍA MESA, M. 2014. Antihypertensive potential of plants used in Cuba. Pharmacology Online 2: 10-17.

GEORGE, G. O. \& F. K. IDU. 2015. Corn silk aqueous extracts and intraocular pressure of systemic and non-systemic hypertensive subjects. Clinical \& Experimental Optometry 98: 138-149. https://doi.org/10.1111/cxo.12240

GÓMEZ, M. A., I. MIGUES, M. CAGGIANIA, X. ARIAS, M. LAPROVITERA, F. BLANCO, M. V. CESIO, E. R. MIGLIAR \& H. HEINZEN. 2016. Vasorelaxant effect of a Baccharis trimera infusion on precontracted rat aortic rings. Natural Products Communications 11: 283-286. https://doi.org/10.1177/1934578X1601100304

GURNI, A. A. 2014. Técnicas histológicas en investigación. En ZARLAVSKY, G. E. (ed.), Histología vegetal. Técnicas simples y complejas, pp. 135-140. Sociedad Argentina de Botánica, Buenos Aires, Argentina.

HARWOKO, S. PRAMONO \& A. E. NUGROHO. 2014. Triterpenoid-rich fraction of Centella asiatica leaves and in vivo antihypertensive activity. International Food Research Journal 21: 149-154.

HOFFMAN, B. B. 2007. Terapéutica de la hipertensión. En GOODMAN, L. S \& A. GILMAN (eds.), Las bases farmacológicas de la terapéutica, pp 845-846. McGraw-Hill Interamericana, México D.F., México.

HURRELL, J. A. 2014. Urban Ethnobotany in Argentina: Theoretical advances and methodological strategies. Ethnobiology and Conservation 3: 2. https://doi.org/10.15451/ec2014-6-3.3-1-11

HURRELL, J. A. 2018. Aloysia citriodora Palau. En ALBUQUERQUE, U. P., U. PATIL \& A. MÁTHÉ (eds.), Medicinal and aromatic plantas of South America, pp. 97-108. Springer, Dordrecht, Netherlands. https://doi.org/10.1007/978-94-024-1552-0 7

HURRELL, J. A., P. M. ARENAS \& I. CRISTINA. 2015a. El conocimiento botánico en zonas urbanas: potenciadores cognitivos comercializados en el Área Metropolitana de Buenos Aires, Argentina. Gaia Scientia 9: 17-31

HURRELL, J. A., J. P. PUENTES \& P. M. ARENAS. 2015b. Medicinal plants with cholesterol-lowering effect marketed in the Buenos Aires-La Plata conurbation, Argentina: An Urban Ethnobotany study. Ethnobiology and Conservation 4: 6. https://doi.org/10.15451/ec2015-7-4.6-1-12

HURRELL, J. A \& M. L. POCHETTINO. 2014. Urban Ethnobotany: theoretical and methodological contributions. En ALBUQUERQUE U. P., L. V. F. CRUZ DA CUNHA, R. F. P. LUCENA \& R. R. N. ALVES (eds), Methods and techniques in Ethnobiology and Ethnoecology, pp. 293-309. Springer, Berlin, Germany. https://doi.org/10.1007/978-1-4614-8636-7_18

HURRELL, J. A. \& J. P. PUENTES. 2013. Medicinal and aromatic species of Asteraceae commercialized in the conurbation Buenos Aires-La Plata (Argentina). Ethnobiology and Conservation 2: 1-40. https://doi.org/10.15451/ec2013-8-2.7-1-40

HURRELL, J. A. \& J. P. PUENTES. 2017. Plant species and products of the Traditional Chinese Phytotherapy in the Ciudad Autónoma de Buenos Aires, Argentina. Ethnobiology and Conservation 6: 1. https://doi.org/10.15451/ec2017-02-6.1-1-43

HURRELL, J. A., M. L. POCHETTINO, J. P. PUENTES \& P. M. ARENAS. 2013. Del marco tradicional al escenario urbano: Plantas ancestrales devenidas suplementos dietéticos en la conurbación Buenos Aires-La Plata, Argentina. Boletín Latinoamericano y del Caribe de Plantas Medicinales y Aromáticas 12: 499-515.

IBARROLA, D., M. C. HELLION \& Y. MONTALBETTI. 2000. Isolation of hypotensive compounds of Solanum sisymbriifolium. Journal of Ethnopharmacology 70: 301-307. https://doi.org/10.1016/S0378-8741(00)00191-4

IBARROLA, D. A., M. C. HELLIÓN-IBARROLA, Y. MONTALBETTI, O. HEINICHEN, M. A. CAMPUZANO, M. L. KENNEDY, N. ALVARENGA, E. A. FERRO, J. H. DÖLZ-VARGAS \& Y. MOMOSE. 2011. Antihypertensive effect of nuatigenin-3-O- $\beta$-chacotriose from Solanum sisymbriifolium Lam. (Solanaceae) (ñuatî pytâ) in experimentally hypertensive (ARH+DOCA) rats under chronic administration. Phytomedicine 8: 634640. https://doi.org/10.1016/j.phymed.2011.01.012

IBODA. 2019. Instituto de Botánica Darwinion. Flora del Cono Sur Catálogo de Plantas Vasculares. Disponible: http://www2.darwin.edu.ar/Proyectos/ FloraArgentina (Consulta: IV-2019).

ICHIMURA, T., A. YAMANAKA, T. ICHIBA, T. TOYOKAWA, Y. KAMADA, T. TAMAMURA \& S. MARUYAMA. 2006. Antihypertensive effect of an extract of Passiflora edulis rind in spontaneously hypertensive rats. Bioscience Biotechnology Biochemestry 70: 718-721. https://doi.org/10.1271/bbb.70.718

INDEC. 2010. Instituto Nacional de Estadística y Censos. Censos Nacionales de Población, Hogares y Viviendas 2001, 2010. Disponible: http://www. indec.gov.ar (Consulta: IV-2019).

KIM, E. Y., I. H BAEK \& M. R. RHYU. 2015. Cardioprotective effects of aqueous Schizandra chinensis fruit extract on ovariectomized and balloon-induced carotid artery injury rat models: 
effects on serum lipid profiles and blood pressure. Journal of Ethnopharmacology 134: 668-675. https://doi.org/10.1016/j.jep.2011.01.019

KONTA, E. M., M. R. ALMEIDA, C. L. DO AMARAL, J. D. DARIN, V. V. DE ROSSO, A. Z. MERCADANTE, L. M. ANTUNES \& M. L. BIANCHI. 2014. Evaluation of the antihypertensive properties of yellow passion fruit pulp (Passiflora edulis Sims f. flavicarpa Deg.) in spontaneously hypertensive rats. Phytotherapy Research 28: 28-32. https://doi.org/10.1002/ptr.4949

KWAN, C. Y., W. B. ZHANG, S. M. SIM, T. DEYAMA $\&$ S. NISHIBE. 2004. Vascular effects of Siberian ginseng (Eleutherococcus senticosus): endotheliumdependent NO- and EDHF-mediated relaxation depending on vessel size. Naunyn Schmiedebergs Archives of Pharmacology 369: 473-480. https://doi.org/10.1007/s00210-004-0927-4

LARA, A., M. ROSAS, G. PASTELÍN, C. AGUILAR, F. ATTIE \& Ó. VELÁZQUEZ MONROY. 2004. Hipercolesterolemia e hipertensión arterial en México: Consolidación urbana actual con obesidad, diabetes y tabaquismo. Archivos de Cardiología de México 74: 220-228.

LAHLOU, S., K. C. TANGI, B. LYOUSSI \& N. MOREL, N. 2008. Vascular effects of Tanacetum vulgare L. leaf extract: in vitro pharmacological study. Journal of Ethnopharmacology 120: 98-102. https://doi.org/10.1016/j.jep.2008.07.041

LEWIS, B. J., K. A. HERRLINGER, T. A. CRAIG, C. E. MEHRING-FRANKLIN, Z. DEFREITAS \& C. HINOJOSA-LABORDE. 2013. Antihypertensive effect of passion fruit peel extract and its major bioactive components following acute supplementation in spontaneously hypertensive rats. The Journal of Nutritional Biochemestry 24: 1359-1366. https://doi.org/10.1016/j.jnutbio.2012.11.003

MARTINS, M. L. L., H. P. PACHECO, I. G. PERINI; D. LENZ; T. U. ANDRADE \& D. C. ENDRINGER. 2013. In vivo hypotensive effect and in vitro inhibitory activity of some Cyperaceae species. Brazilian Journal of Pharmaceutical Sciences 49: 803-809.

https://doi.org/10.1590/S1984-82502013000400020

MELIS, M. S. 1995. Chronic administration of aqueous extract of Stevia rebaudiana in rats: renal effects. Journal of Ethnopharmacology 47: 129-134. https://doi.org/10.1016/0378-8741(95)01271-E

MICUCCI, M., A. ANGELETTI, M. CONT, I. CORAZZA, R. ALDINI, E. DONADIO, A. CHIARINI \& R. BUDRIESI. 2016. Hibiscus sabdariffa L. flowers and Olea Europea L. leaves extract-based formulation for hypertension care: in vitro efficacy and toxicological profile. Journal of Medicinal Food 19: 504-512. https://doi.org/10.1089/jmf.2015.0072
MIRANDA, J. J., J. C. K. WELLS \& L. SMEETH. 2012. Transiciones en contexto: hallazgos vinculados con la migración rural-urbana en enfermedades no transmisibles en Perú. Revista Peruana de Medicina Experimental y Salud Pública 29: 366-372. https://oi.org/10.1590/S1726-46342012000300012

MOGHADAMTOUSI, S. Z., M. FADAEINASAB, S. NIKZAD, G. MOHAN, H. M. ALI \& H. A. KADIR. 2015. Annona muricata (Annonaceae): A review of its traditional uses, isolated acetogenins and biological activities. International Journal of Molecular Sciences 16: 15625-15658. https://doi.org/10.3390/ijms160715625

MOHAMED, H., A. MOHAMED, A. Z. NAOUFEL \& E. MOHAMED. 2016. Pharmacological Evidence of $\alpha$-adrenergic Receptors in the Hypotensive Effect of Chamaemulum nobile L. Cardiovascular \& Hematological Agents in Medicinal Chemistry (Formerly Current Medicinal ChemistryCardiovascular \& Hematological Agents) 14: 53-58. https://doi.org/10.2174/187152571401160613190635

MOMTAZI-BOROJENI, A. A., S. A., ESMAEILI, E. ABDOLLAHI \& A. S. AHEBKAR. 2017. A Review on the Pharmacology and Toxicology of Steviol Glycosides Extracted from Stevia rebaudiana. Current Pharmaceutical Design 23: 1616-1622. https://doi.org/10.2174/1381612822666161021142835

MORADI, M. T., M., ASADI-SAMANI \& M. BAHMANI. 2016. Hypotensive medicinal plants according to ethnobotanical evidence of Iran: A systematic review. International Journal of Pharm Tech Research 9: 416-426.

MOLARES, S., P. M. ARENAS \& A. AGUILAR. 2012. Etnobotánica urbana de los productos vegetales adelgazantes comercializados en México DF. Boletín Latinoamericano y del Caribe de Plantas Medicinales y Aromáticas 11: 400-412.

MOREIRA, F. V., J. F. A. BASTOS, A. F. BLANK, P. B. ALVES, M. R. \& V. SANTOS. 2010. Chemical composition and cardiovascular effects induced by the essential oil of Cymbopogon citratus DC. Stapf, Poaceae, in rats. Revista Brasileira de Farmacognosia 20: 904-909. https://doi.org/10.1590/S0102-695X2010005000012

NANSY, E., HARWOKO, S. PRAMONO \& A. E. NUGROHO. 2015. Total flavonoid content and in vivo hypotensive effect of chloroform insoluble fraction of Centella asiatica leaf extract. International Food Research Journal 22: 2119 2125 .

NWOKOCHA, C. R., D. U. OWU, A. GORDON, K. THAXTER, G. MCCALLA, R. I. OZOLUA \& L. YOUNG. 2012. Possible mechanisms of action of the hypotensive effect of Annona muricata (soursop) in normotensive Sprague-Dawley rats. Pharmaceutical Biology 50: 1436-1441. https://doi.org/10.3109/13880209.2012.684690 
NWOKOCHA, C. R., R. I. OZOLUA, D. U. OWU, M. I. NWOKOCHA \& A. C. UGWU. 2011. Antihypertensive properties of Allium sativum (garlic) on normotensive and two kidney one clip hypertensive rats. Nigerian Journal of Physiological Sciences 26: 213-238.

OJEWOLE, J. A. 2005. Hypoglycemic and hypotensive effects of Psidium guajava Linn. (Myrtaceae) leaf aqueous extract. Methods and Findings in Experimental and Clinical Pharmacology 27: 689-95. https://doi.org/10.1358/mf.2005.27.10.948917

OMS. 2013. Información general de la hipertensión en el mundo. Disponible: https://www.who.int/ cardiovascular_diseases/publications/global_brief_ hypertension/es/ (Consulta: IV-2019).

OMS. 2019. Organización Mundial de la Salud. Hipertensión. Disponible: https://www.who.int/ topics/hypertension/es/ (Consulta: IV-2019).

PÉREZ, J. H. \& A. P. UNANUA. 2002. Hipertensión arterial. Everest, $160 \mathrm{pp}$.

POCHETTINO, M. L., J. P. PUENTES, F. BUET COSTANTINO, P. M. ARENAS, E. A. ULIBARRI \& J. A. HURRELL. 2012. Functional foods and nutraceuticals in a market of Bolivian immigrants in Buenos Aires (Argentina). Evidence-Based Complementary and Alternative Medicine 2012: 320193. https://doi.org/10.1155/2012/320193

POCHETTINO, M. L. \& J. A. HURRELL. 2013. Saberes y plantas en las diagonales: transmisión del conocimiento botánico urbano. Boletín de la Sociedad Argentina de Botánica 48 (Supl.): 16.

PUENTES, J. P. 2016. Plantas medicinales y productos derivados comercializados como antidiabéticos en la conurbación Buenos Aires-La Plata, Argentina. Boletín Latinoamericano del Caribe de Plantas Medicinales y Aromáticas 15: 373 - 397.

PUENTES, J. P. 2017. Etnobotánica urbana: El conocimiento botánico local sobre las plantas alimenticias y medicinales, y sus usos, en la conurbación Buenos Aires-La Plata (Argentina). Tesis doctoral, Universidad Nacional de La Plata, Argentina. 300 pp.

PUENTES, J. P. \& J. A. HURRELL. 2015. Plantas andinas y sus productos comercializados con fines medicinales y alimentarios en el Área Metropolitana Buenos Aires- La Plata, Argentina. Boletín Latinoamericano y del Caribe de Plantas Medicinales y Aromáticas 14: 206-236.

QUINLAN, M. 2005. Considerations for collecting free lists in the field: examples from Ethnobotany. Field Methods 17: 1-16. https://doi.org/10.1177/1525822X05277460

RAGONE, M. I., M. STELlA, A. PASTORE \& A. E. CONSOLINI. 2010. Sedative and cardiovascular effects of Aloysia citriodora Palau on mice and rats. Latin American Journal of Pharmacy 29: 79-86.
RANILLA, L. G., Y. I. KWON, E. APOSTOLIDIS \& K. SHETTY. 2010. Phenolic compounds, antioxidant activity and in vitro inhibitory potential against key enzymes relevant for hyperglycemia and hypertension of commonly used medicinal plants, herbs and spices in Latin America. Bioresource Technology 101: 4676-4689.

https://doi.org/10.1016/j.biortech.2010.01.093

ROGHANI-DEHKORDI, F. \& A. F. KAMKHAH. 2009.

Artichoke leaf juice contains antihypertensive effect in patients with mild hypertension. Journal of Dietary Supplements 6: 328-341. https://doi.org/10.3109/19390210903280207

SINGH, V. K. \& D. K. SINGH. 2008. Pharmacological Effects of Garlic (Allium sativum L.). Annual Review of Biomedical Sciences 10.

https://doi.org/10.5016/1806-8774.2008.v10p6

SOLÀ, R., E. BRUCKERT, R. M. VALLS, S. NAREJOS, X. LUQUE, M. CASTRO-CABEZAS, G. DOMÉNECH, F. TORRES, M. HERAS, X. FARRÉS, J. V. VAQUER, J. M. MARTÍNEZ, M. C. ALMARAZ \& A. ANGUERA A. 2010. Soluble fibre (Plantago ovata Husk) reduces plasma lowdensity lipotrein (LDL) colesterol, triglycerides, insulin, oxidised LDL and systolic blood pressure in hypercholesterolaemic patients: A randomised trial. Atherosclerosis 211: 630-637.

https://doi.org/10.1016/j.atherosclerosis.2010.03.010

TEIXEIRA, K., P. DOS SANTOS, V. CITADINI ZANETTE, S. DALBÓ \& P. A. AMARAL. 2017. Medicinal Plants that can Cause Changes in Blood Pressure and Interactions with Antihypertensive Agents. American Journal of Ethnomedicine 4: 2. https://doi.org/10.21767/2348-9502.100002

TEVES, M. R., P. H. PACHECO, C. BAZÁN. \& G. H. WENDEL. 2018. Diuretic activity of methanolic extracts from Jodina rhombifolia aerial parts on Wistar rats. Journal of Pharmacy \& Pharmacognosy Research 6: 402-411.

THE PLANT LIST. 2013. Version 1.1. Disponible: http://www.theplantlist.org/ (Consulta: IV-2019).

TROPICOS.ORG. Missouri Botanical Garden. Disponible: http://www.tropicos.org (Consulta: IV-2019).

ULBRICHT, C., W. CHAO, K. NUMMY, E. RUSIE, S. TANGUAY-COLUCCI, C. M. IANNUZZI, J. B. PLAMMOOTTIL, M. VARGHESE \& W. WEISSNER. 2009. Chia (Salvia hispanica): A systematic review by the natural standard research collaboration. Reviews on Recent Clinical Trials 4: 168-174. https://doi.org/10.2174/157488709789957709

ULLAH, R., M. NADEEM, A. KHALIQUE, M. IMRAN, S. MEHMOOD, A. JAVID \& J. HUSSAIN. 2016. Nutritional and therapeutic perspectives of Chia (Salvia hispanica L.): A review. Journal of Food Science Technology 53: 1750-1758. https://oi.org/10.1007/s13197-015-1967-0 
VEIGA, V. F., A. C. PINTO \& M. A. M. MACIEL. 2005. Plantas medicinais: Cura segura? Quimica Nova 3: 519-528.

https://doi.org/10.1590/S0100-40422005000300026

WIGATI, D., K. ANWAR, SUDARSONO \& A. E. NUGROHO. 2017. Hypotensive activity of ethanolic extracts of Morinda citrifolia L. leaves and fruit in dexamethasone-induced hypertensive rat. Journal of Evidence-Based Complementary Alternative Medicine 22: 107-113.

https://doi.org/10.1177/2156587216653660
YIN, M.H., D.G. KANG, D.H. CHOI, T.O. KWON \& H.S. LEE.2005. Screening of vasorelaxant activity of some medicinal plants used in Oriental medicines. Journal of Ethnopharmacology 99 :113117. https://doi.org/10.1016/j.jep.2005.02.013

ZHANG, X., X. YANG, Y. LIN, M. SUO, L. GONG, J. CHEN \& R. HUI. 2015. Anti-hypertensive effect of Lycium barbarum $\mathrm{L}$. with down-regulated expression of renal endothelial lncRNA sONE in a rat model of salt-sensitive hypertension. International Journal of Clinical and Experimental Pathology 8: 6981-6987. 
